\title{
NONUNIFORMLY ELLIPTIC EQUATIONS: POSITIVITY OF WEAK SOLUTIONS
}

\author{
BY C. V. COFFMAN ${ }^{1}$, R. J. DUFFIN ${ }^{2}$ AND V. J. MIZEL $^{3}$ \\ Communicated by Philip Hartman, August 17, 1972
}

1. This note is concerned with the weak boundary value problem

$$
\int_{\Omega}\left(\sum_{i, j=1}^{N} a_{i j}(x) u_{x_{i}} v_{x_{i}}+b(x) u v\right) d x=\int_{\Omega} c(x) f v d x, \text { all } v \in C_{0}^{\infty}(\Omega),
$$

and the weak eigenvalue problem

$$
\text { (2) } \int_{\Omega}\left(\sum_{i, j=1}^{N} a_{i j}(x) u_{x_{i}} v_{x_{i}}+b(x) u v\right) d x=\lambda \int_{\Omega} c(x) u v d x \text {, all } v \in C_{0}^{\infty}(\Omega) \text {, }
$$

where $\Omega$ is a connected open set in $R^{N}$. Our hypothesis concerning the coefficient matrix $\left(a_{i j}\right)$ in (1) and (2) is similar to but weaker than those imposed on the elliptic operators which are studied in [2], [3], [4]. Specifically, we assume that $A=\left(a_{i j}\right)$ is a real matrix-valued function, symmetric and positive definite almost everywhere on $\Omega$ with

$$
\|A\|,\left\|A^{-1}\right\| \in L_{\mathrm{loc}}^{1}(\Omega) \text {. }
$$

Concerning the coefficients $b, c$ our assumptions are the following: $b$ and $c$ are real valued,

$$
M b \geqq c>0 \quad \text { a.e. on } \Omega
$$

for some positive constant $M$ and

$$
b, b^{-1}, c \in L_{\mathrm{loc}}^{1}(\Omega) \text {. }
$$

Under these assumptions we prove: If $f \in L^{2}(\Omega, c(x) d x), f(x) \geqq 0$ a.e. on $\Omega$ and $f \neq 0$ then (1) has a solution positive almost everywhere on $\Omega$, in particular a nonnegative eigenfunction of (2) is positive almost everywhere in $\Omega$; if (2) has a nonnegative eigenfunction corresponding to an eigenvalue $\lambda_{1}>0$ then $\lambda_{1}$ is simple and the spectrum of (2) is contained in the interval $\left[\lambda_{1}, \infty\right]$.

This research was motivated by certain problems arising in connection with the study in [1] of nonlinear elliptic eigenvalue problems.

AMS (MOS) subject classifications (1970). Primary 35J20, 35J25.

Key words and phrases. Nonuniformly elliptic, positive solutions, eigenvalue problem, boundary value problem.

${ }^{1}$ Research partially supported by National Science Foundation under grant GP-21512.

2 Research partially supported by Army Research Office (Durham) under research grant DA-AROD-31-124-71-G17.

${ }^{3}$ Research partially supported by the National Science Foundation under grant GP-28377. 
2. We assume that $\left(a_{i j}\right), b$ and $c$ are as above. Let $\dot{X}_{1}$ denote the set of functions $u \in C^{\infty}(\Omega)$ for which

$$
\|u\|_{X_{1}}^{2}=\int_{\Omega}\left(\sum_{i, j=1}^{N} a_{i j} u_{x_{i}} u_{x_{j}}+b u^{2}\right) d x<\infty,
$$

and let $X_{1}$ be the Hilbert space obtained by completing $X_{1}$ in the norm (6); $X_{0}$ will denote the closure of $C_{0}^{\infty}(\Omega)$ in $X_{1}$.

LEMMA 1. The space $X_{1}$ is stronger than the space $H_{\text {loc }}^{1,1}(\Omega)$, and the norm on $X_{1}$ is given by (6). The spaces $X_{1}$ and $X_{0}$ are closed under the operation

$$
u \rightarrow|u|
$$

moreover, this operation is norm preserving in $X_{1}$.

Here, as is standard, $H_{\text {loc }}^{1,1}(\Omega)$ denotes the Fréchet space of locally integrable, locally strongly $L^{1}$ differentiable functions on $\Omega$.

Let $X$ be any closed subspace of $X_{1}$ with

$$
X_{0} \subset X \subset X_{1},
$$

and such that $X$ is closed under the mapping (7), and let $Y$ denote the Hilbert space consisting of measurable functions $f$ for which

$$
\|f\|_{y}^{2}=\int_{\Omega}|f|^{2} c(x) d x<\infty .
$$

From (4) and (6) it is clear that the functions in $X$ are also in $Y$. The inclusion mapping $X \subset Y$ will be denoted by $i$.

LEMMA 2. The mapping $i: X \rightarrow Y$ is bounded, injective, and has dense range. The mapping $i^{*}: Y \rightarrow X$ (the Lax-Milgram operator) is also injective with dense range and preserves nonnegativity.

It is not difficult to see that when $X=X_{0}$ then $u=i^{*} f$, for $f \in Y$, is the solution of (1).

We next consider the "Green's operator" $k=i i^{*}$ in $Y$, and state the first of our two main results which refines the nonnegativity assertion of Lemma 2.

THEOREM 1. The operator $k$ is selfadjoint, positive definite, and bounded. If $f$ is a nonzero element of $Y$ and $f(x) \geqq 0$ a.e. on $\Omega$ then $h=k f$ satisfies

$$
h(x)>0 \text { a.e. on } \Omega \text {. }
$$

In particular if $k$ has a nonnegative eigenfunction $\varphi$, then

$$
\varphi(x)>0 \text { a.e. on } \Omega \text {. }
$$


REMARK. If $\Omega$ is bounded, $b=0$, and the coefficients in (2) satisfy stronger regularity conditions then such a positivity result can be obtained from Lemma 2 and the Harnack inequality of Trudinger [4]; indeed in that case one can assert, instead of merely (9), that $h$ has a positive essential lower bound on each compact subset of $\Omega$. Our proof of Theorem 1 however makes use of global rather than local methods.

THEOREM 2. Let $\varphi$ be a nonnegative eigenfunction of $k, \mu \varphi=k \varphi$, then $\|k\|=\mu$, and $\mu$ is a simple eigenvalue of $k$.

While Theorem 2 is very easily proved in the case where $k$ is compact, the general case is somewhat deeper and does not seem to be contained in the extensive literature on positive operators.

3. We now describe the sort of application of Theorems 1 and 2 which was wanted for $[\mathbf{1}]$. We consider the problem $(2)$ in $W_{0}^{1, p}(\Omega)$ for some $p$ with $2 \leqq p \leqq \infty$. With $p$ fixed we take

$$
r=p /(p-2)
$$

and we take $s$ to be an element of the extended real number system with $p \leqq s$ and $s \leqq N p /(N-p)$, if $p \leqq N$, finally we take

$$
r_{1}=s /(s-2) \text {. }
$$

We assume that the matrix $A$ is as in $\$ 1$ and in addition that

$$
\|A\| \in L^{r}(\Omega) \text {. }
$$

Concerning $b$ and $c$ we assume only that

$$
b, c \in L^{r_{1}}(\Omega) \text {. }
$$

THEOREM 3. Let $u \in W_{0}^{1, p}(\Omega)$ be a nonnegative eigenfunction of the weak problem (2) corresponding to the eigenvalue $\lambda_{1}>0$. Then

$$
u(x)>0 \text { a.e. in } \Omega
$$

and, for all $v \in W_{0}^{1, p}(\Omega)$,

$$
\int_{\Omega}\left(\sum_{i, j=1}^{N} a_{i j} v_{x_{i}} v_{x_{j}}+b v^{2}\right) d x \geqq \lambda_{1} \int_{\Omega} v^{2} c(x) d x,
$$

with equality only if $v$ is proportional to $u$.

\section{REFERENCES}

1. C. V. Coffman, Existence and uniqueness of positive eigenfunctions for a class of quasilinear elliptic boundary value problems of sublinear type, Indiana Univ. Math. J. (to appear).

2. S. N. Kružkov, Certain properties of solutions to elliptic equations, Dokl. Akad. Nauk SSSR 150 (1963), 470-473 = Soviet Math. Dokl. 4 (1963), 686-695. MR 27 \#440. 
3. M. K. V. Murthy and G. Stampacchia, Boundary value problems for some degenerateelliptic operators, Ann. Mat. Pure Appl. (4) 80 (1968), 1-122. MR 40 \# 3069.

4. N. Trudinger, On the regularity of generalized solutions of linear non-uniformly elliptic equations, Arch. Rational Mech. Anal. 42 (1971), 50-62.

Department of Mathematics, Carnegie-Mellon University, Pittsburgh, PennsylVANIA 15123 